\title{
Computing the NMR Spectrum of a Bulk Ionic Liquid Phase by QM/MM Methods
}

\author{
Alessandro Bagno, * Fabio D’Amico and Giacomo Saielli \\ Supporting Information
}

\section{Complete ref. 25}

Gaussian 03, Revision C.02, Frisch, M. J.; Trucks, G. W.; Schlegel, H. B.; Scuseria, G. E.; Robb, M. A.; Cheeseman, J. R.; Montgomery, Jr., J. A.; Vreven, T.; Kudin, K. N.; Burant, J. C.; Millam, J. M.; Iyengar, S. S.; Tomasi, J.; Barone, V.; Mennucci, B.; Cossi, M.; Scalmani, G.; Rega, N.; Petersson, G. A.; Nakatsuji, H.; Hada, M.; Ehara, M.; Toyota, K.; Fukuda, R.; Hasegawa, J.; Ishida, M.; Nakajima, T.; Honda, Y.; Kitao, O.; Nakai, H.; Klene, M.; Li, X.; Knox, J. E.; Hratchian, H. P.; Cross, J. B.; Bakken, V.; Adamo, C.; Jaramillo, J.; Gomperts, R.; Stratmann, R. E.; Yazyev, O.; Austin, A. J.; Cammi, R.; Pomelli, C.; Ochterski, J. W.; Ayala, P. Y.; Morokuma, K.; Voth, G. A.; Salvador, P.; Dannenberg, J. J.; Zakrzewski, V. G.; Dapprich, S.; Daniels, A. D.; Strain, M. C.; Farkas, O.; Malick, D. K.; Rabuck, A. D.; Raghavachari, K.; Foresman, J. B.; Ortiz, J. V.; Cui, Q.; Baboul, A. G.; Clifford, S.; Cioslowski, J.; Stefanov, B. B.; Liu, G.; Liashenko, A.; Piskorz, P.; Komaromi, I.; Martin, R. L.; Fox, D. J.; Keith, T.; Al-Laham, M. A.; Peng, C. Y.; Nanayakkara, A.; Challacombe, M.; Gill, P. M. W.; Johnson, B.; Chen, W.; Wong, M. W.; Gonzalez, C.; and Pople, J. A.; Gaussian, Inc., Wallingford CT, 2004.

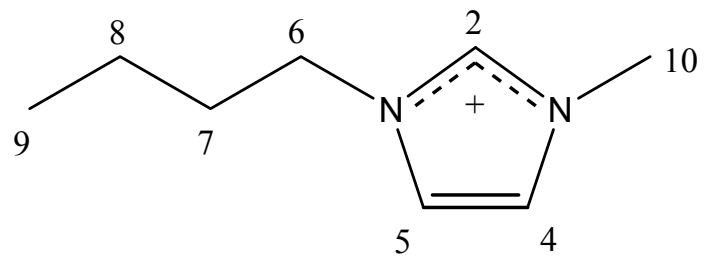

Scheme S1. Structural formula and numbering of the 1-butyl-3-methylimidazolium cation [bmim]. 

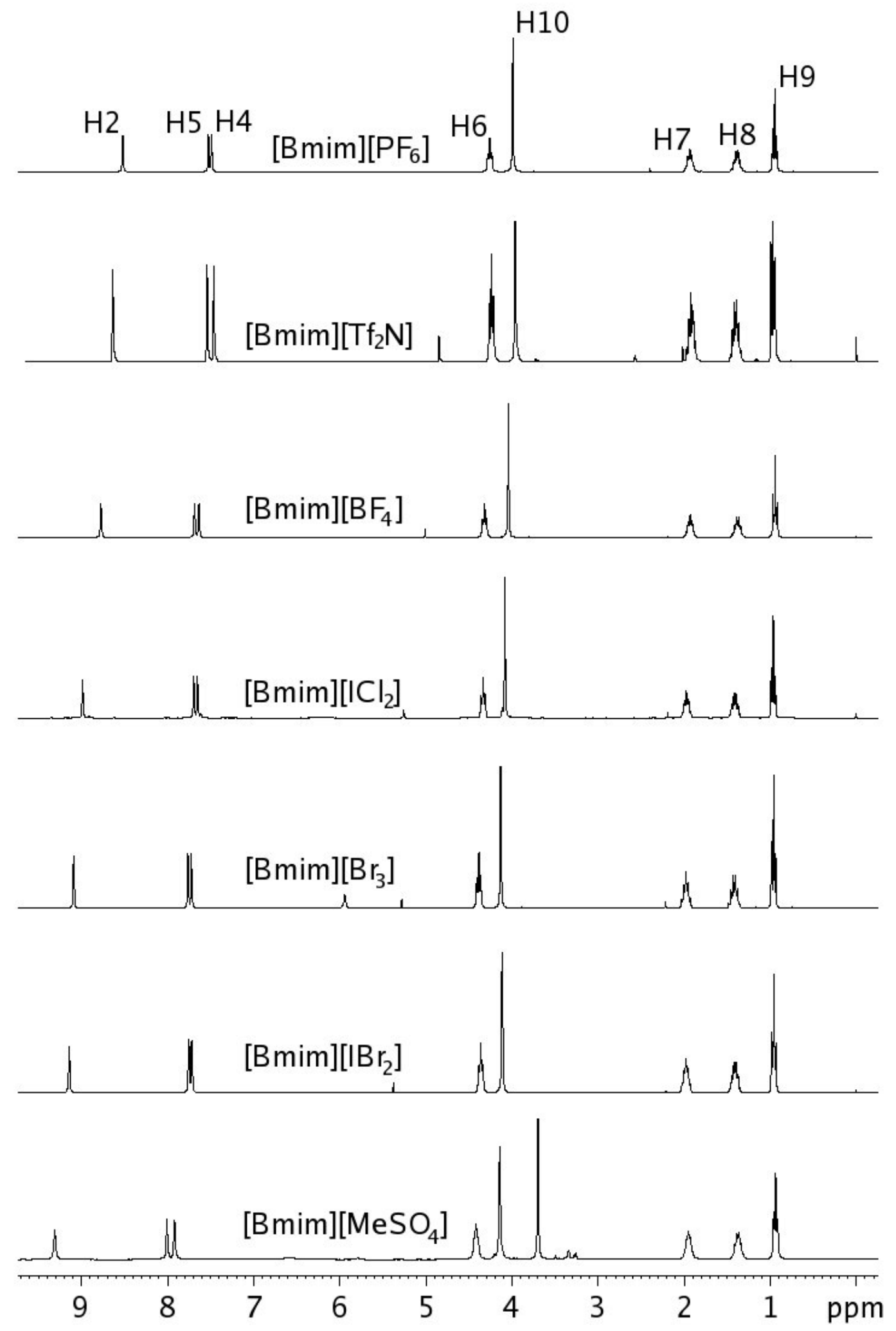

Figure S1. ${ }^{1}$ H NMR spectra of [bmim]-based ILs. See Table S1 for chemical shifts. 

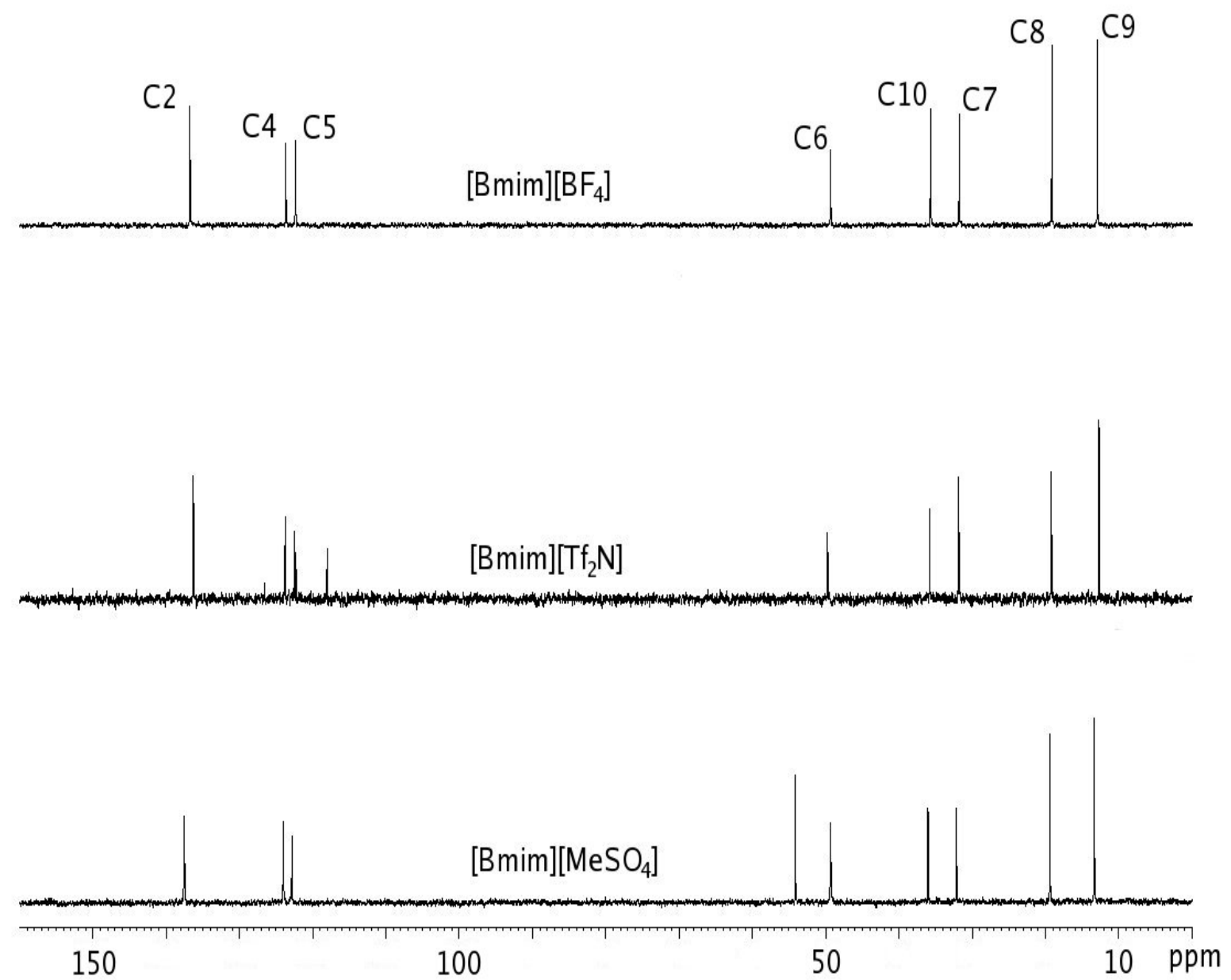

Figure S2. ${ }^{13} \mathrm{C}$ NMR spectra of [bmim]-based ILs. See Table S1 for chemical shifts. 
Table S1. Experimental ${ }^{1} \mathrm{H}$ and ${ }^{13} \mathrm{C}$ chemical shifts of [bmim]-based ionic liquids. ${ }^{a}$

\begin{tabular}{cccccccccccc}
\hline & \multicolumn{1}{c}{$\delta\left({ }^{1} \mathrm{H}\right) / \mathrm{ppm}$} \\
\cline { 2 - 28 } & {$\left[\mathrm{PF}_{6}\right]$} & {$\left[\mathrm{Tf}_{2} \mathrm{~N}\right]$} & {$\left[\mathrm{BF}_{4}\right]$} & {$\left[\mathrm{ICl}_{2}\right]$} & {$\left[\mathrm{Br}_{3}\right]$} & {$\left[\mathrm{IBr}_{2}\right]$} & {$\left[\mathrm{MeSO}_{4}\right]$} & & {$\left[\mathrm{Tf}_{2} \mathrm{~N}\right]$} & {$\left[\mathrm{BF}_{4}\right]$} & {$\left[\mathrm{MeSO}_{4}\right]$} \\
$\mathrm{H} 2$ & 8.52 & 8.63 & 8.77 & 8.97 & 9.09 & 9.14 & 9.31 & $\mathrm{C} 2$ & 136.2 & 136.8 & 137.6 \\
$\mathrm{H} 5$ & 7.52 & 7.54 & 7.69 & 7.68 & 7.75 & 7.75 & 8.00 & $\mathrm{C} 4$ & 122.5 & 122.4 & 122.9 \\
$\mathrm{H} 4$ & 7.49 & 7.46 & 7.63 & 7.74 & 7.72 & 7.71 & 7.92 & $\mathrm{C} 5$ & 123.7 & 123.7 & 124.1 \\
$\mathrm{H} 6$ & 4.25 & 4.24 & 4.32 & 4.32 & 4.33 & 4.36 & 4.42 & $\mathrm{C} 6$ & 49.7 & 49.3 & 49.4 \\
$\mathrm{H} 10$ & 3.97 & 3.96 & 4.04 & 4.07 & 4.13 & 4.11 & 4.14 & $\mathrm{C} 10$ & 35.8 & 35.7 & 36.1 \\
$\mathrm{H} 7$ & 1.93 & 1.92 & 1.93 & 1.96 & 1.98 & 1.97 & 1.95 & $\mathrm{C} 7$ & 31.8 & 31.7 & 32.3 \\
$\mathrm{H} 8$ & 1.38 & 1.40 & 1.38 & 1.40 & 1.42 & 1.40 & 1.37 & $\mathrm{C} 8$ & 19.2 & 19.1 & 19.5 \\
$\mathrm{H} 9$ & 0.94 & 0.97 & 0.94 & 0.95 & 0.96 & 0.95 & 0.93 & $\mathrm{C} 9$ & 12.7 & 12.9 & 13.5 \\
$\mathrm{MSO}_{4}$ & & & & & & & 3.70 & $\mathrm{MeSO}_{4}$ & & & 54.2 \\
\hline
\end{tabular}

${ }^{\mathrm{a}}$ Reference: internal TMS. Data for [ $\left[\mathrm{ICl}_{2}\right],\left[\mathrm{Br}_{3}\right]$ and $\left[\mathrm{IBr}_{2}\right]$ from ref. 16.

Table S2. ${ }^{1} \mathrm{H}$ chemical shifts of [bmim] calculated at the DFT level for the isolated [bmim] cation and for the four ion pairs shown in Figure S3. ${ }^{a}$

\begin{tabular}{ccccccccccc}
\hline & \multicolumn{3}{c}{$\delta\left({ }^{1} \mathrm{H}\right) / \mathrm{ppm}$} \\
\cline { 2 - 11 } & Isolated & {$\left[\mathrm{PF}_{6}\right]$} & {$\left[\mathrm{Tf}_{2} \mathrm{~N}\right]$} & {$\left[\mathrm{BF}_{4}\right]$} & {$\left[\mathrm{MeSO}_{4}\right]$} & & Isolated & {$\left[\mathrm{Tf}_{2} \mathrm{~N}\right]$} & {$\left[\mathrm{BF}_{4}\right]$} & {$\left[\mathrm{MeSO}_{4}\right]$} \\
$\mathrm{H} 2$ & 7.66 & 10.66 & 12.47 & 13.91 & 12.25 & $\mathrm{C} 2$ & 138.1 & 149.0 & 156.5 & 153.2 \\
$\mathrm{H} 5$ & 7.33 & 6.96 & 7.02 & 6.47 & 6.83 & $\mathrm{C} 4$ & 131.1 & 126.9 & 124.0 & 125.9 \\
$\mathrm{H} 4$ & 7.27 & 6.91 & 6.94 & 6.34 & 6.74 & $\mathrm{C} 5$ & 130.5 & 126.3 & 125.2 & 124.8 \\
$\mathrm{H} 6$ & 3.97 & 4.05 & 4.64 & 3.86 & 3.87 & $\mathrm{C} 6$ & 58.6 & 56.2 & 56.6 & 56.3 \\
$\mathrm{H} 10$ & 3.81 & 4.06 & 4.34 & 3.76 & 4.07 & $\mathrm{C} 10$ & 38.8 & 38.8 & 37.2 & 37.2 \\
$\mathrm{H} 7$ & 1.64 & 2.10 & 1.73 & 1.69 & 1.87 & $\mathrm{C} 7$ & 41.8 & 41.5 & 38.2 & 38.1 \\
$\mathrm{H} 8$ & 1.39 & 1.43 & 1.57 & 1.07 & 1.24 & $\mathrm{C} 8$ & 26.2 & 24.6 & 25.3 & 25.8 \\
$\mathrm{H} 9$ & 1.04 & 1.08 & 1.01 & 0.60 & 0.88 & $\mathrm{C} 9$ & 15.7 & 15.7 & 15.9 & 15.0 \\
$\mathrm{MeSO}_{4}$ & & & & & 3.29 & $\mathrm{MeSO}_{4}$ & & & & 54.2 \\
\hline
\end{tabular}

${ }^{\mathrm{a}}$ Chemical shifts are referred to TMS calculated at the same level of theory. 

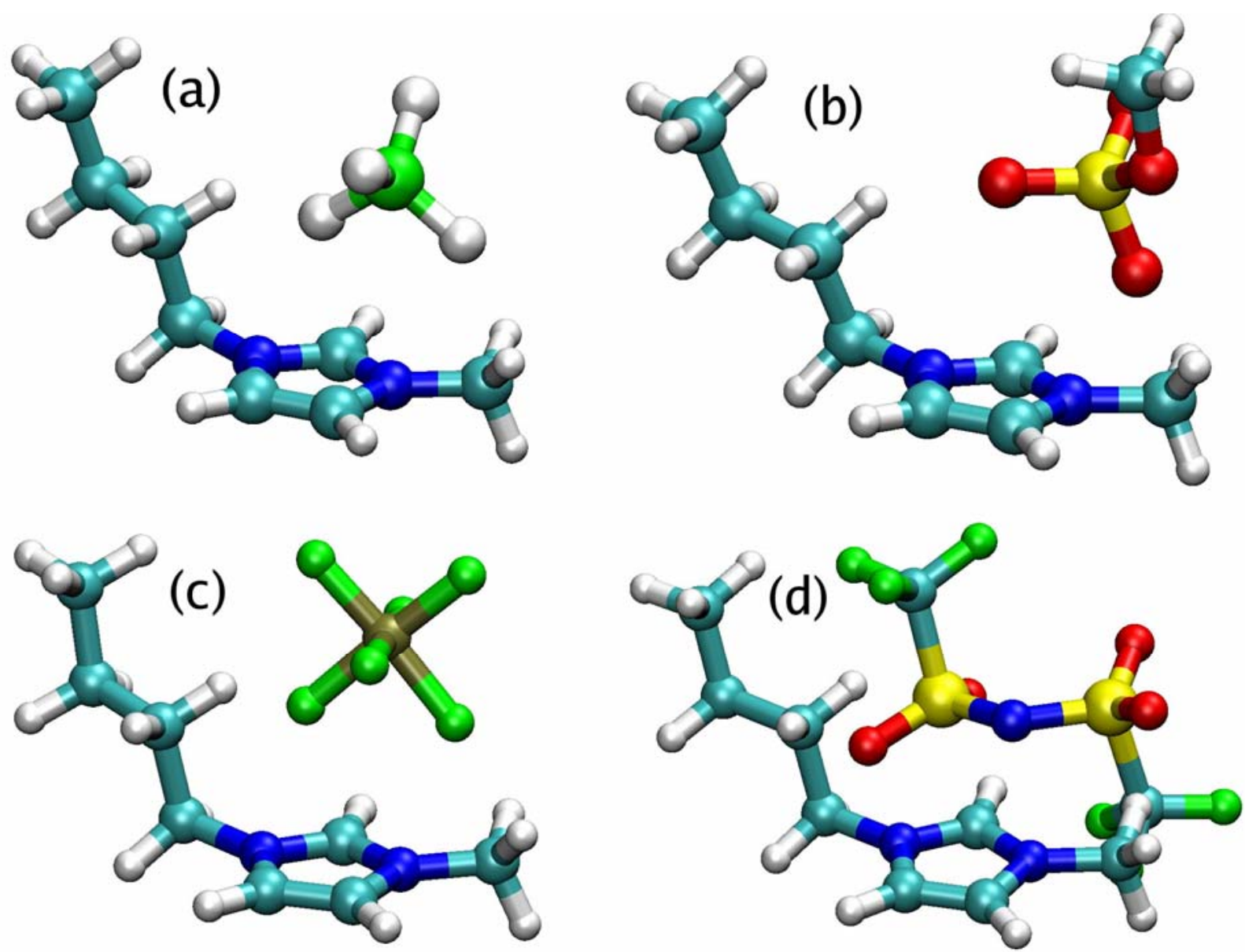

Figure S3. Optimized geometry (B3LYP/6-31G(d,p)) in vacuum of the ion pairs investigated by DFT calculations: (a) $[\mathrm{bmim}]\left[\mathrm{BF}_{4}\right],(\mathrm{b})[\mathrm{bmim}]\left[\mathrm{MeSO}_{4}\right],(\mathrm{c})[\mathrm{bmim}]\left[\mathrm{PF}_{6}\right],(\mathrm{d})[\mathrm{bmim}]\left[\mathrm{Tf}_{2} \mathrm{~N}\right]$. Some geometrical parameters are reported in Table S3.

Table S3. Distance $R$ between $\mathrm{C} 2$ of [bmim] and the central atom of the anion (B in $\left[\mathrm{BF}_{4}\right], \mathrm{P}$ in $\left[\mathrm{PF}_{6}\right], \mathrm{S}$ in $\left[\mathrm{MeSO}_{4}\right]$ and $\mathrm{N}$ in $\left[\mathrm{Tf}_{2} \mathrm{~N}\right]$ ), and angle $\alpha \mathrm{C} 2-\mathrm{H} 2$-central atom of the anion.

\begin{tabular}{ccccc}
\hline & {$\left[\mathrm{BF}_{4}\right]$} & {$\left[\mathrm{Tf}_{2} \mathrm{~N}\right]$} & {$\left[\mathrm{MeSO}_{4}\right]$} & {$\left[\mathrm{PF}_{6}\right]$} \\
\hline $\mathrm{R} / \AA$ & 3.078 & 3.048 & 3.335 & 3.525 \\
$\alpha / \operatorname{deg}$ & 121.5 & 176.1 & 127.3 & 129.9 \\
\hline
\end{tabular}



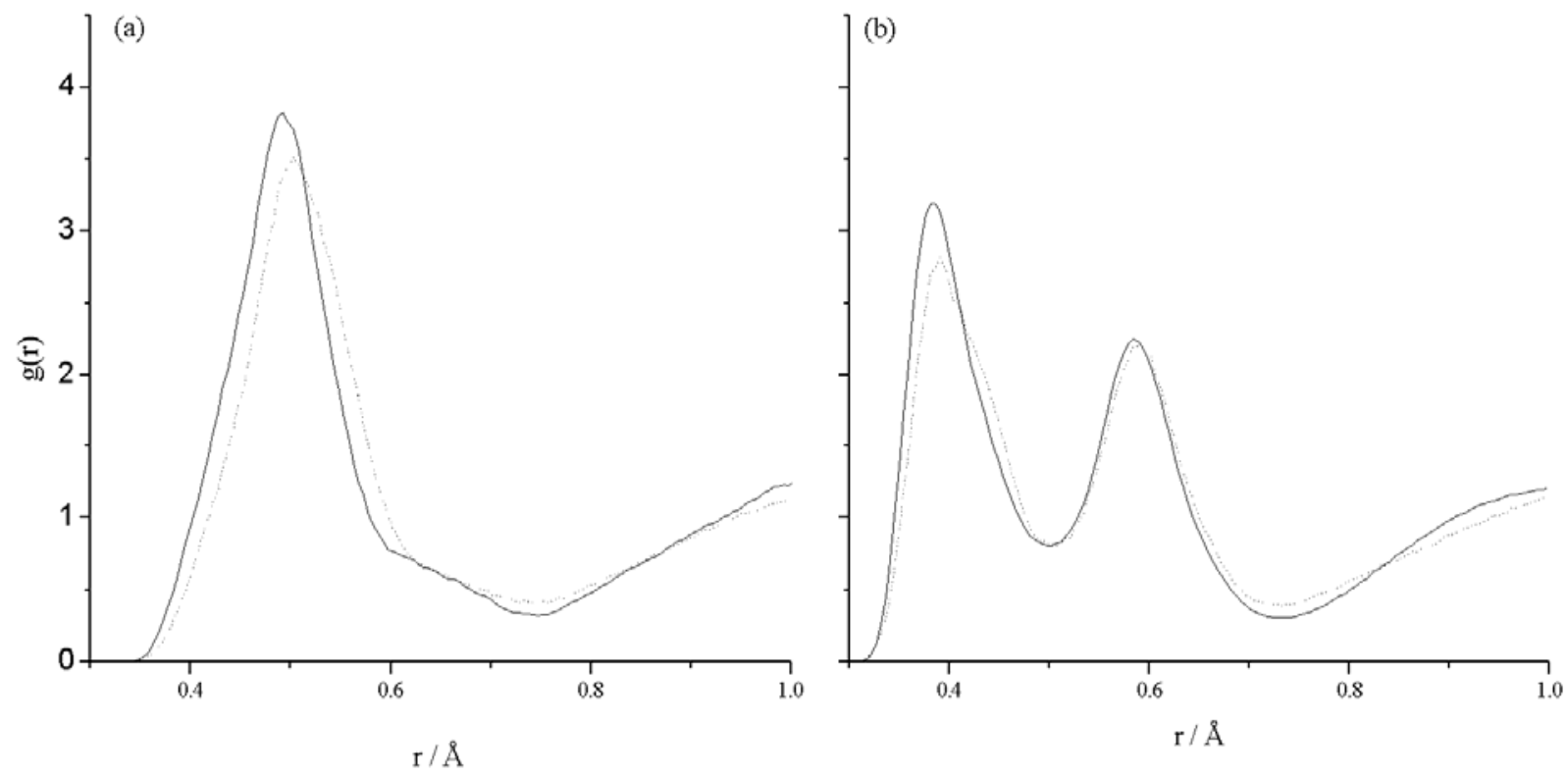

Figure S4. (A) Radial distribution function, $g(r)$, between the centre of the imidazolium ring and the centre of mass of the anion for $[\mathrm{bmim}]\left[\mathrm{BF}_{4}\right]$ (solid) and $[\mathrm{bmim}]\left[\mathrm{MeSO}_{4}\right]$ (dotted). The broad peak at about $5 \AA$ corresponds to four anions after integration up to $5.7 \AA$. (B) Radial distribution function, $g(r)$, between carbon $\mathrm{C} 2$ and the centre of mass of the anion for $[\mathrm{bmim}]\left[\mathrm{BF}_{4}\right]$ (solid) and $[\mathrm{bmim}]\left[\mathrm{MeSO}_{4}\right]$ (dotted). The first peak, having a maximum at about $3.8 \AA$, integrates to two anions.

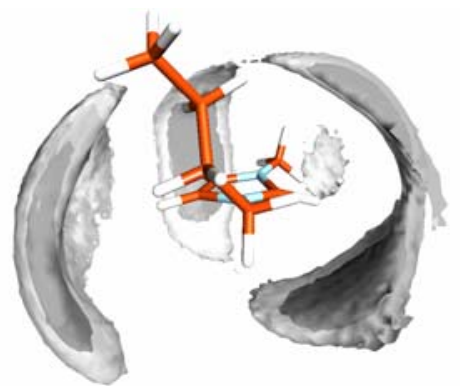

(a)

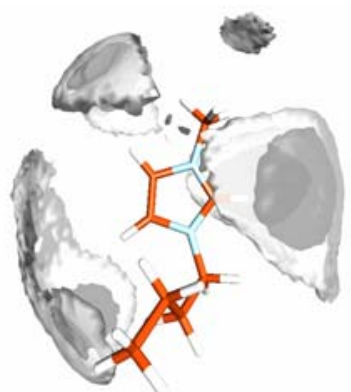

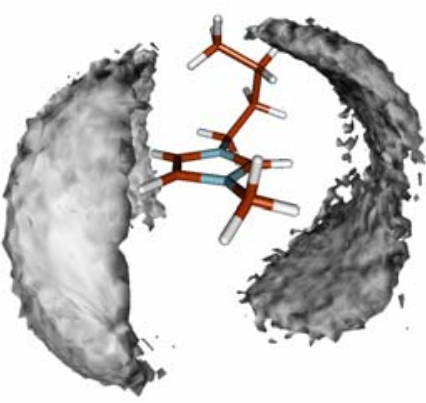

(b)

Figure S5. Spatial distribution function $(\mathrm{SDF})$ of anions around [bmim]. (a) $\left[\mathrm{BF}_{4}\right]$. Left: top view; right: side view. Light and dark grey isodensity contours correspond to a probability density of 2.5 and 9.9, respectively.(b) $\left[\mathrm{MeSO}_{4}\right]$. Isodensity contour is for a probability density of 6.2 . 
Table S4. Calculated ${ }^{1} \mathrm{H}$ chemical shifts (ppm) at the (A)-(E) QM/MM levels. ${ }^{\text {a }}$

\begin{tabular}{cccccccc}
\hline \multicolumn{9}{c}{$\left[\mathrm{BF}_{4}\right]$} & \multicolumn{3}{c}{$\left[\mathrm{MeSO}_{4}\right]$} \\
\hline & $(\mathrm{A})$ & $(\mathrm{B})$ & $(\mathrm{C})$ & $(\mathrm{D})$ & $(\mathrm{E})$ & $(\mathrm{C})$ & $(\mathrm{E})$ \\
$\mathrm{H} 2$ & 10.95 & 10.20 & 9.83 & 10.06 & 9.48 & 11.36 & 11.00 \\
$\mathrm{H} 5$ & 10.69 & 9.68 & 9.36 & 9.53 & 8.55 & 10.89 & 9.76 \\
$\mathrm{H} 4$ & 10.59 & 8.91 & 9.63 & 9.65 & 8.81 & 11.00 & 9.94 \\
$\mathrm{H} 6$ & 7.46 & 6.43 & 6.15 & 6.30 & 5.43 & 7.62 & 6.69 \\
$\mathrm{H} 10$ & 7.23 & 5.80 & 6.05 & 6.11 & 5.23 & 7.43 & 6.52 \\
$\mathrm{H} 7$ & 5.17 & 3.95 & 3.84 & 3.98 & 3.03 & 5.29 & 4.28 \\
$\mathrm{H} 8$ & 4.61 & 3.37 & 3.33 & 3.46 & 2.57 & 4.68 & 3.76 \\
$\mathrm{H} 9$ & 4.38 & 2.82 & 2.83 & 3.09 & 2.03 & 4.19 & 3.16 \\
\hline
\end{tabular}

a H and L stand for high (QM, B3LYP/cc-pVTZ) and low (MM, point charges) layers, respectively. [bmim] is always in the QM layer. (A): [bmim] only (i.e. conformationally averaged); (B), (C), (D): surrounding ions (L) up to a cutoff of $6.0,7.8$ or $10.0 \AA$ from the centre of the imidazolium ring; (E): the two anions closest to $\mathrm{C} 2$ (H) plus surrounding ions (L) up to a cutoff of $7.8 \AA$. Chemical shifts are referred to TMS as described in the text.

Table S5. Calculated ${ }^{13} \mathrm{C}$ chemical shifts (ppm) at the (A)-(E) QM/MM levels. ${ }^{\text {a }}$

\begin{tabular}{cccccccc}
\hline \multicolumn{9}{c}{$\left[\mathrm{BF}_{4}\right]$} & \multicolumn{4}{c}{$\left[\mathrm{MeSO}_{4}\right]$} \\
\hline & $(\mathrm{A})$ & $(\mathrm{B})$ & $(\mathrm{C})$ & $(\mathrm{D})$ & $(\mathrm{E})$ & $(\mathrm{C})$ & $(\mathrm{D})$ \\
$\mathrm{C} 2$ & 132.26 & 136.52 & 133.97 & 134.67 & 135.43 & 134.86 & 136.43 \\
$\mathrm{C} 4$ & 125.75 & 119.74 & 127.01 & 125.48 & 125.81 & 125.70 & 125.14 \\
$\mathrm{C} 5$ & 125.14 & 124.37 & 121.34 & 122.12 & 120.71 & 122.82 & 121.98 \\
$\mathrm{C} 6$ & 49.89 & 46.80 & 46.96 & 47.69 & 47.05 & 46.97 & 46.71 \\
$\mathrm{C} 10$ & 33.86 & 31.82 & 33.84 & 33.73 & 34.60 & 34.00 & 34.98 \\
$\mathrm{C} 7$ & 31.18 & 28.54 & 29.98 & 30.20 & 29.74 & 29.57 & 29.87 \\
C8 & 20.92 & 18.71 & 19.60 & 20.14 & 19.57 & 19.87 & 20.00 \\
$\mathrm{C} 9$ & 10.98 & 10.00 & 10.83 & 10.66 & 10.50 & 10.59 & 11.07 \\
\hline
\end{tabular}

${ }^{\mathrm{a}} \mathrm{See}$ footnote to Table S4.

Table S6. Calculated $\sigma_{\mathrm{TMS}}(\mathrm{ppm})$ at the (A)-(E) QM/MM levels. ${ }^{\mathrm{a}}$

\begin{tabular}{cccccccc}
\hline \multicolumn{9}{c}{$\left[\mathrm{BF}_{4}\right]$} \\
\hline & $(\mathrm{A})$ & $(\mathrm{B})$ & $(\mathrm{C})$ & $(\mathrm{D})$ & $(\mathrm{E})$ & $(\mathrm{C})$ & $(\mathrm{E})$ \\
${ }^{1} \mathrm{H}$ & 34.9431 & 33.6807 & 33.4143 & 33.7900 & 32.8699 & 34.6274 & 34.0096 \\
${ }^{13} \mathrm{C}$ & 180.7527 & 179.2171 & 180.1170 & 180.0814 & 179.6829 & 180.4170 & 180.1722 \\
\hline
\end{tabular}

${ }^{\mathrm{a}}$ See footnote to Table S4. 


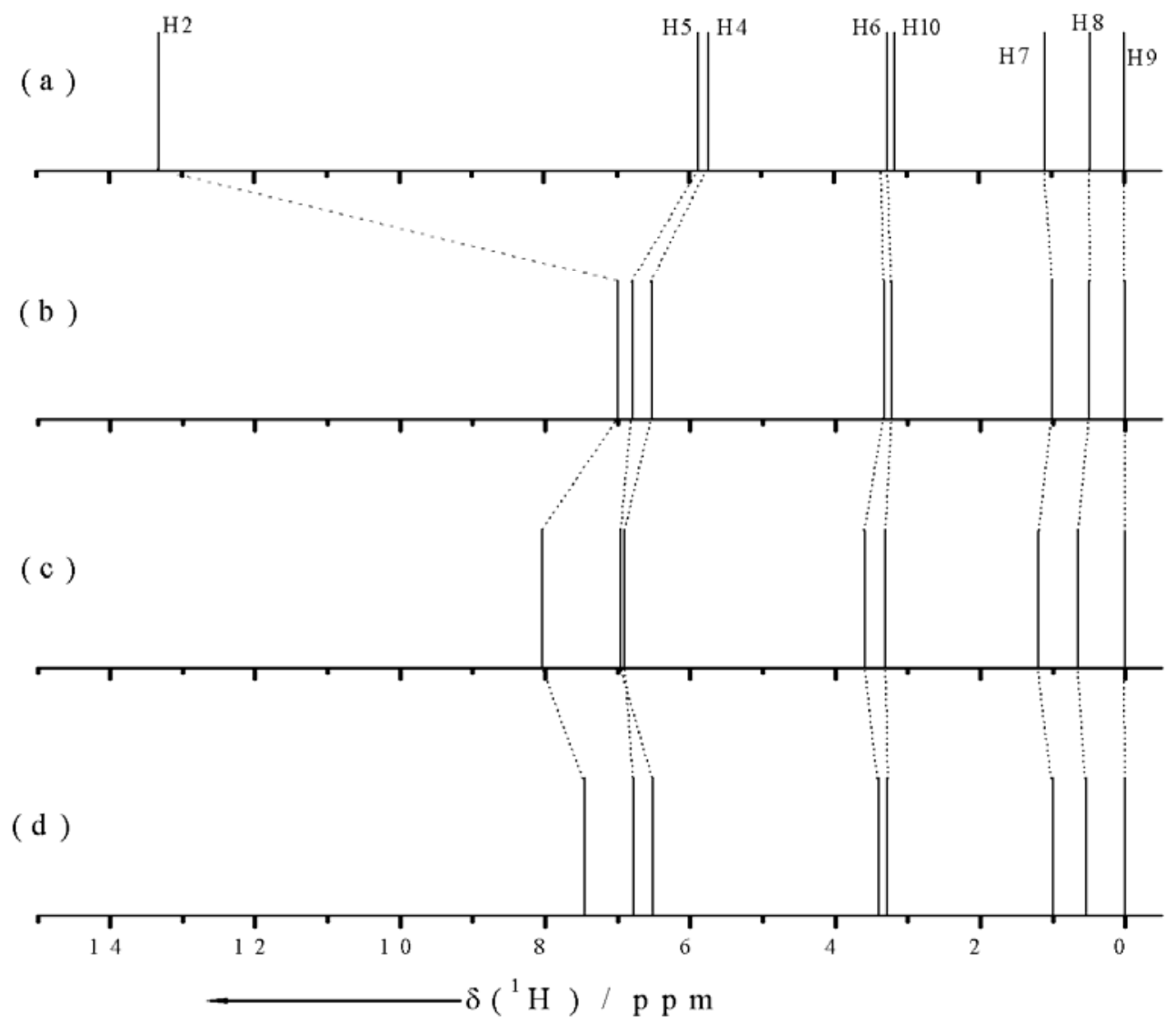

Figure S6. ${ }^{1} \mathrm{H}$ stick spectra of $[\mathrm{bmim}]\left[\mathrm{BF}_{4}\right]$. (a) Ion pair; (b) level (C); (c) experimental; (d) level (E). Dotted lines connect equivalent sites. Chemical shifts are referred to H9. 


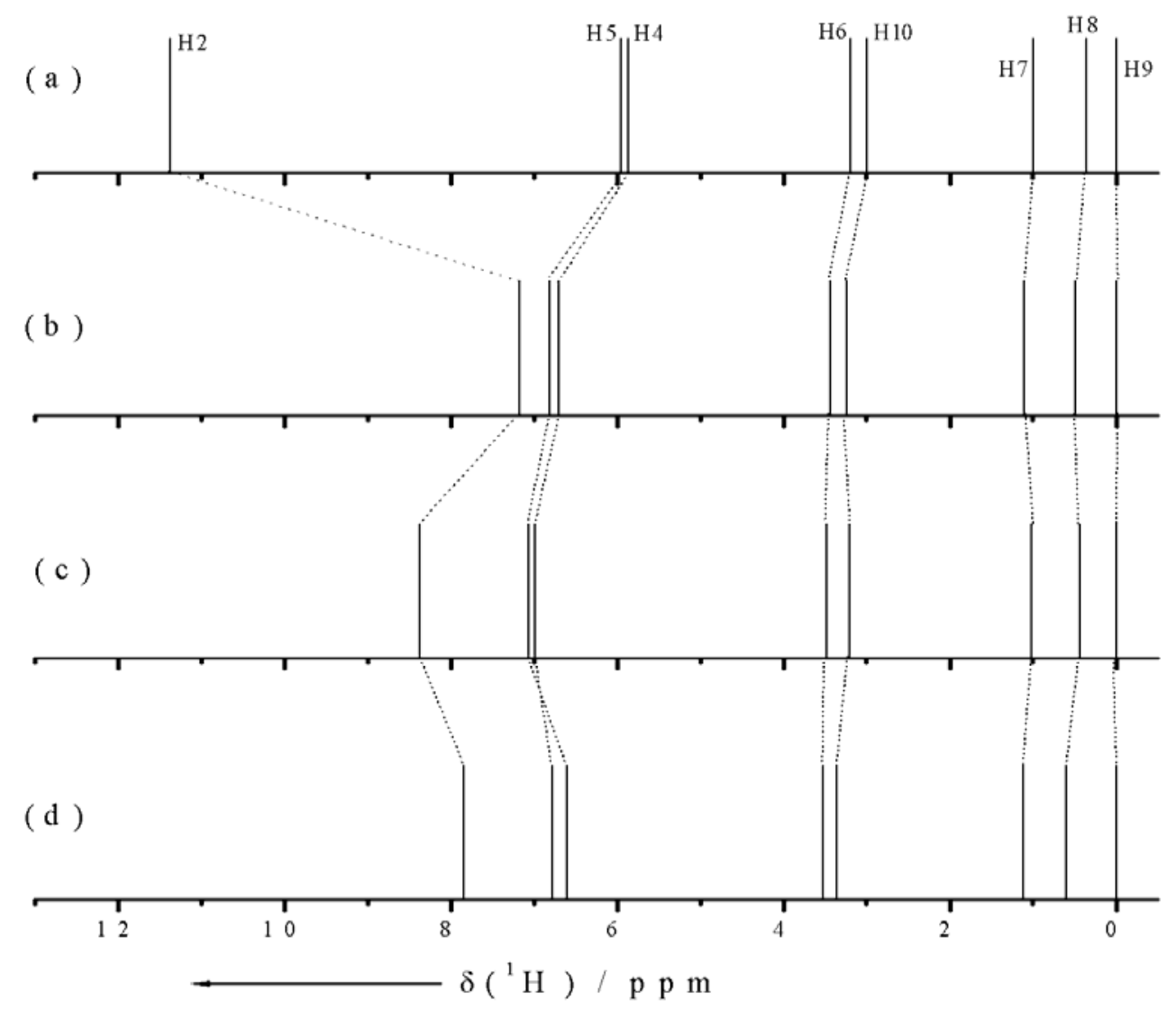

Figure S7. ${ }^{1} \mathrm{H}$ stick spectra of [bmim] $\left[\mathrm{MeSO}_{4}\right]$.(a) Ion pair; (b) level (C); (c) experimental; (d) level (E). Dotted lines connect equivalent sites. Chemical shifts are referred to H9. 


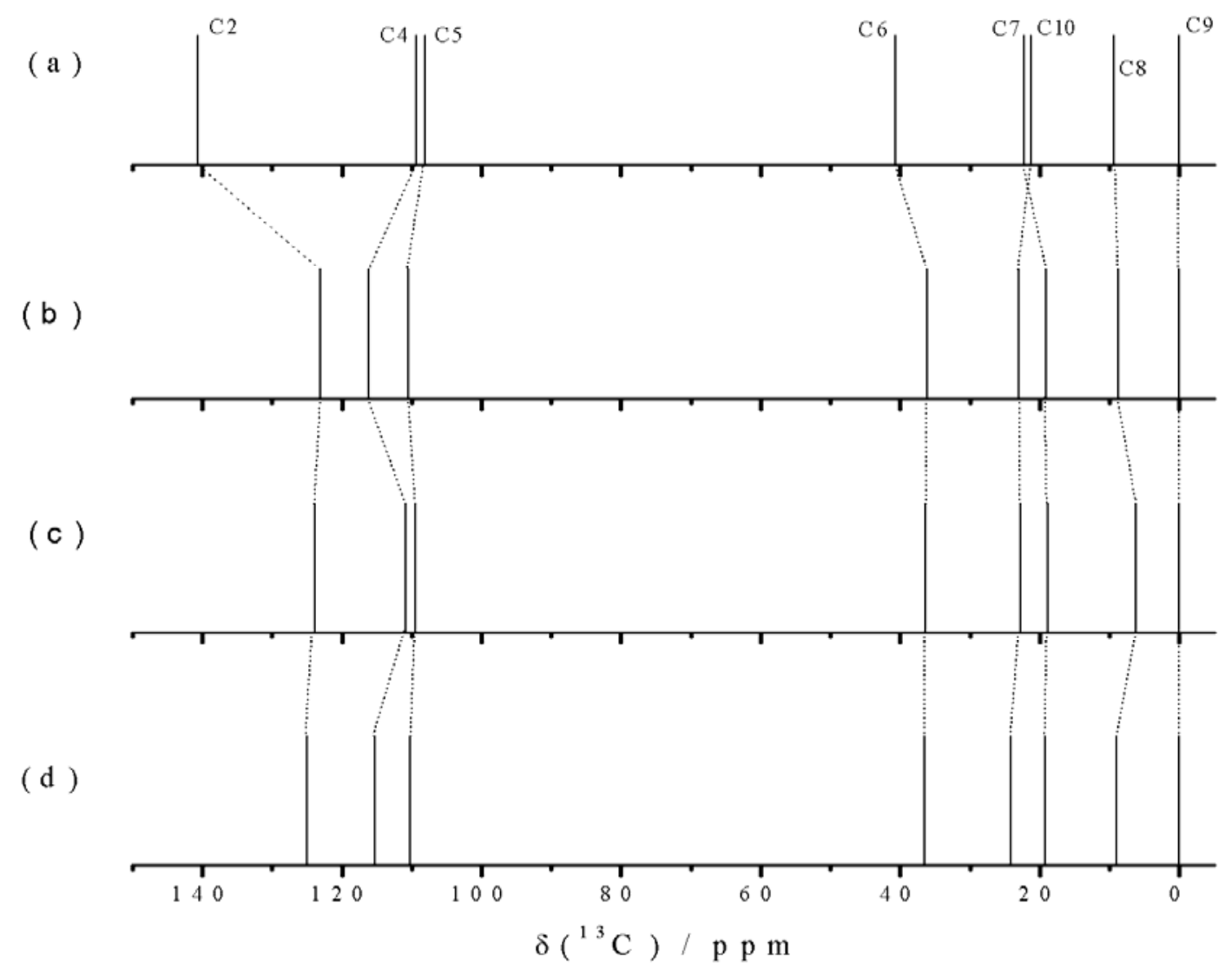

Figure S8. ${ }^{13} \mathrm{C}$ stick spectra of [bmim] $\left[\mathrm{BF}_{4}\right]$.(a) Ion pair; (b) level (C); (c) experimental; (d) level (E). Dotted lines connect equivalent sites. Chemical shifts are referred to $\mathrm{C} 9$. 
(a)

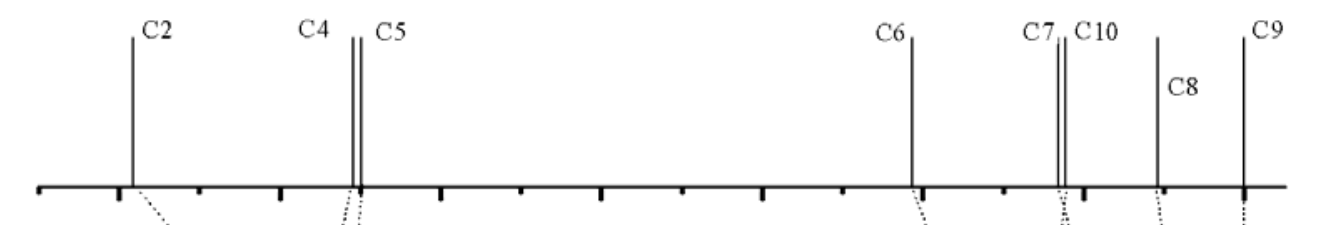

( b )

(c)

(d)

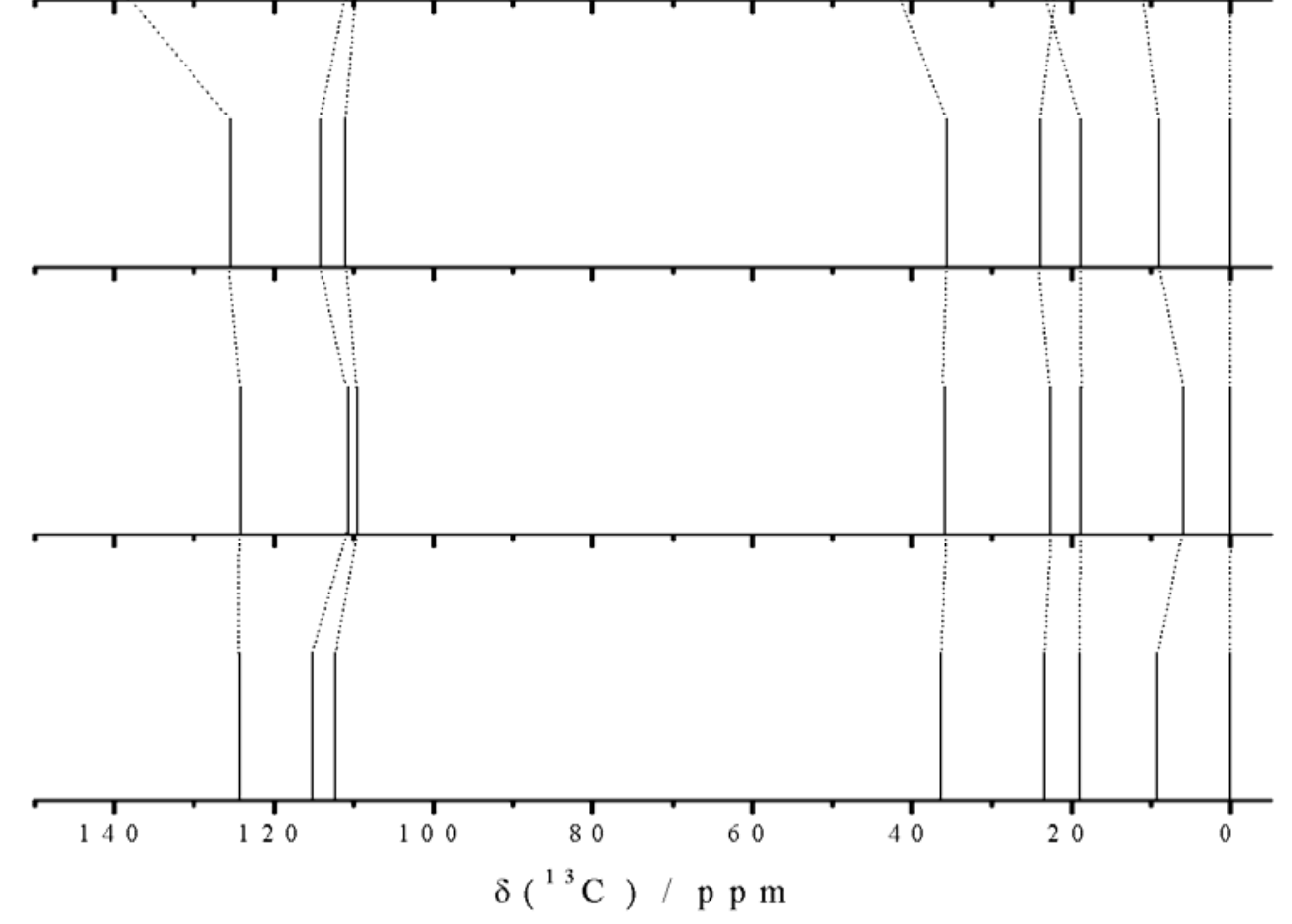

Figure S9. ${ }^{13} \mathrm{C}$ stick spectra of $[\mathrm{bmim}]\left[\mathrm{MeSO}_{4}\right]$.(a) Ion pair; (b) level (C); (c) experimental; (d) level (E). Dotted lines connect equivalent sites. Chemical shifts are referred to $\mathrm{C} 9$. 


\section{Cartesian coordinates of isolated [bmim] and ion pairs B3LYP/6-31G(d,p) geometry}

\section{[bmim]}

$\begin{array}{rrrr}\mathrm{C} & -2.395013 & -0.848762 & -0.612035 \\ \mathrm{~N} & -2.483026 & -0.095361 & 0.543909 \\ \mathrm{C} & -1.076422 & -1.141235 & -0.799482 \\ \mathrm{C} & -1.249042 & 0.060740 & 1.040856 \\ \mathrm{~N} & -0.376274 & -0.567260 & 0.244878 \\ \mathrm{C} & -3.721762 & 0.432641 & 1.132589 \\ \mathrm{H} & -4.214969 & 1.088155 & 0.413156 \\ \mathrm{H} & -4.381915 & -0.395028 & 1.396964 \\ \mathrm{H} & -3.474681 & 1.000183 & 2.029174 \\ \mathrm{C} & 1.095627 & -0.588172 & 0.419418 \\ \mathrm{C} & 1.810126 & 0.426092 & -0.478357 \\ \mathrm{C} & 3.333242 & 0.375228 & -0.286206 \\ \mathrm{C} & 4.066888 & 1.389059 & -1.169021 \\ \mathrm{H} & 5.146716 & 1.329644 & -1.010900 \\ \mathrm{H} & 3.875611 & 1.204909 & -2.231335 \\ \mathrm{H} & 3.753142 & 2.413544 & -0.943615 \\ \mathrm{H} & 3.572860 & 0.563678 & 0.768339 \\ \mathrm{H} & 3.693603 & -0.637321 & -0.508637 \\ \mathrm{H} & 1.561704 & 0.223675 & -1.527756 \\ \mathrm{H} & 1.437173 & 1.433075 & -0.253344 \\ \mathrm{H} & 1.291502 & -0.391808 & 1.476647 \\ \mathrm{H} & 1.423300 & -1.609635 & 0.209217 \\ \mathrm{H} & -1.000001 & 0.602208 & 1.940382 \\ \mathrm{H} & -3.265576 & -1.113644 & -1.191100 \\ \mathrm{H} & -0.585226 & -1.706838 & -1.575281\end{array}$




\section{[bmim] $\left[\mathrm{BF}_{4}\right]$}

$\begin{array}{lrrr}\mathrm{C} & -2.218896 & -2.234774 & -0.590624 \\ \mathrm{~N} & -2.397960 & -0.953422 & -0.096431 \\ \mathrm{C} & -0.973815 & -2.636588 & -0.208304 \\ \mathrm{C} & -1.296742 & -0.607188 & 0.560101 \\ \mathrm{~N} & -0.417334 & -1.598696 & 0.519790 \\ \mathrm{C} & -3.539757 & -0.059146 & -0.316749 \\ \mathrm{H} & -3.881926 & -0.173198 & -1.344707 \\ \mathrm{H} & -4.352104 & -0.303728 & 0.371760 \\ \mathrm{H} & -3.185976 & 0.967953 & -0.156846 \\ \mathrm{C} & 0.982901 & -1.474847 & 0.977757 \\ \mathrm{C} & 1.928806 & -1.158473 & -0.185360 \\ \mathrm{C} & 3.313125 & -0.739593 & 0.324990 \\ \mathrm{C} & 4.300898 & -0.476352 & -0.814854 \\ \mathrm{H} & 5.273631 & -0.157278 & -0.428074 \\ \mathrm{H} & 4.461399 & -1.376084 & -1.420002 \\ \mathrm{H} & 3.930394 & 0.311625 & -1.478471 \\ \mathrm{H} & 3.190731 & 0.170734 & 0.923874 \\ \mathrm{H} & 3.719914 & -1.510960 & 0.994497 \\ \mathrm{H} & 2.012729 & -2.032382 & -0.846047 \\ \mathrm{H} & 1.487374 & -0.339009 & -0.760348 \\ \mathrm{H} & 0.993649 & -0.647088 & 1.690323 \\ \mathrm{H} & 1.247294 & -2.402194 & 1.494949 \\ \mathrm{H} & -1.100483 & 0.508854 & 1.092891 \\ \mathrm{H} & -2.974501 & -2.736261 & -1.160963 \\ \mathrm{H} & -0.444421 & -3.552868 & -0.391741 \\ \mathrm{~B} & -0.116298 & 2.175271 & -0.020525 \\ \mathrm{~F} & 0.395646 & 3.414109 & -0.292848 \\ \mathrm{~F} & 0.577228 & 1.548038 & 1.063001 \\ \mathrm{~F} & -0.041372 & 1.303265 & -1.131564 \\ \mathrm{~F} & -1.492352 & 2.252356 & 0.374296\end{array}$


[bmim][MeSO 4 ]

\begin{tabular}{|c|c|c|c|}
\hline C & -2.999600 & 0.418410 & 1.316140 \\
\hline $\mathrm{N}$ & -2.090110 & 1.305670 & 0.765941 \\
\hline $\mathrm{C}$ & -2.279670 & -0.513234 & 2.001760 \\
\hline C & -0.855121 & 0.925300 & 1.110670 \\
\hline $\mathrm{N}$ & -0.943015 & -0.176982 & 1.862040 \\
\hline C & -2.378960 & 2.445540 & -0.113849 \\
\hline $\mathrm{H}$ & -1.433350 & 2.776110 & -0.548350 \\
\hline $\mathrm{H}$ & -2.849210 & 3.247290 & 0.460125 \\
\hline $\mathrm{H}$ & -3.045690 & 2.116060 & -0.911879 \\
\hline C & 0.218755 & -0.966466 & 2.312720 \\
\hline C & 0.371848 & -2.272210 & 1.528390 \\
\hline C & 1.618890 & -3.046630 & 1.972720 \\
\hline $\mathrm{C}$ & 1.793830 & -4.362500 & 1.209230 \\
\hline $\mathrm{H}$ & 2.691090 & -4.894330 & 1.540020 \\
\hline $\mathrm{H}$ & 1.890290 & -4.181130 & 0.133993 \\
\hline $\mathrm{H}$ & 0.936746 & -5.028560 & 1.361270 \\
\hline $\mathrm{H}$ & 1.567540 & -3.250590 & 3.051570 \\
\hline $\mathrm{H}$ & 2.501930 & -2.414990 & 1.815450 \\
\hline $\mathrm{H}$ & 0.450674 & -2.015460 & 0.467364 \\
\hline $\mathrm{H}$ & -0.522224 & -2.895810 & 1.666300 \\
\hline $\mathrm{H}$ & 0.102079 & -1.145820 & 3.386630 \\
\hline $\mathrm{H}$ & 1.094970 & -0.335644 & 2.155580 \\
\hline $\mathrm{H}$ & 0.046007 & 1.428150 & 0.761769 \\
\hline $\mathrm{H}$ & -4.062740 & 0.524130 & 1.173180 \\
\hline $\mathrm{H}$ & -2.598830 & -1.374320 & 2.565860 \\
\hline S & 1.200060 & 1.149420 & -1.507030 \\
\hline O & 0.834574 & 2.357680 & -0.710262 \\
\hline O & 1.256470 & -0.080462 & -0.666960 \\
\hline 0 & 2.293140 & 1.312220 & -2.468200 \\
\hline O & -0.210313 & 0.972349 & -2.412660 \\
\hline C & -0.135068 & -0.048036 & -3.410950 \\
\hline $\mathrm{H}$ & -1.096370 & -0.042218 & -3.931260 \\
\hline $\mathrm{H}$ & 0.029476 & -1.032050 & -2.955830 \\
\hline $\mathrm{H}$ & 0.670879 & 0.161367 & -4.120870 \\
\hline
\end{tabular}

